\title{
Parameter Estimation of Low Intensity Wireless Network Receiving Signal Spectrum
}

\author{
Youwei Shao $^{1}$ \\ ${ }^{1}$ School of Applied Electronics, Chongqing College of Electronic Engineering, \\ Chongqing, China \\ E-mail: cqshaoyouwei@126.com
}

\begin{abstract}
For subnanosecond low intensity of wireless network signal with representative Beidou Satellite navigation signal is difficult to obtain accurate time estimation and Angle estimation in the positioning, and easy to be affected by environmental noise, which made its positioning accuracy not higher, this paper proposed a parameter estimation method based on low intensity wireless network receiving signal spectrum. By sampling approach, first sampling launch signal as multidimensional independent sub-signal and modeling independently, through constructing noise space and sub-single space to obtain TOA estimation accurately based on the orthogonalization of corresponding column vector; then using complex domain mapping, on the basis of obtained TOA estimation, to obtain DOA estimation with contrast way accurately. Finally, it conducted accuracy analysis of the proposed parameter estimation method. Test data showed that: compared with PM algorithm, ESPRIT algorithm, the proposed technique was more accurate on ${ }_{T O A}$ and DOA estimation; and in case of low strength of signal and serious background noise, the proposed method can still be effective to maintain the precision of parameters estimation. The technology can effectively reduce the influence of background noise on the signal transmission, has strong practical deployment significance.
\end{abstract}

Keywords: Wireless network; Receiving signal spectrum; DOA estimation; TOA estimation; Complex mapping

\section{Introduction}

In the process of low intensity wireless signal positioning, the time of arrive estimation (TOA estimation)of signal and the direction of time of arrival of signal wave estimation (DOA estimation) can be acquired accurately, which have become the most important two influencing parameters [1-3] of satellite positioning accuracy. This paper proposed a new receiving spectrum parameter estimation mechanism. It first conducts sampling the received signals to get multi-channel independent sub-signals. For better improving antinoise performance at the same time, according to the property of the sub signal at the receiving process, based on the characteristic value and characteristic vector, the signal space is divided into mutual independent signal subspace and noise subspace, and the orthogonalization precise separation of signal and noise was realized, and the influence of noise on positioning accuracy was reduced. At the same time, according to the characteristics of mutual orthogonal signal subspace and noise subspace to carry on complex domain mapping, the TOA and DOA estimation were accurately calculated; And the TOA and DOA estimation problems were evolved into the problem on zero solving on complex domain, and the accurate precision calculation and error control in the complex domain was realized; finally, simulation analysis was carried on the proposed method. 


\section{Signal Model}

Assumed that the low intensity of wireless network signal based on nanosecond through the space-based platform to launch, the launch baseband signal using hexadecimal frequency-shift keying (16FSK modulation), the signal after modulation is divided into $M$ sub-signal to transmit, sub-signal of each path is divided into $\lambda$ signal transmission paths. Its unit impulse response $H^{(k)}(\omega)$ is:

$$
H^{(M)}(\omega)=\sum_{l=1}^{\lambda} \beta_{l}^{(M)} \varepsilon\left(\omega-\omega_{l}\right)
$$

Where, $\varepsilon(\omega)$ represents unit step response, $\beta_{l}^{(M)}$ represents the frequency domain signal fading of $M$ th sub-single at $l$ th path, $\omega_{l}$ represents the frequency delay of the frequency domain signal on this path.

On the basis of model (1), then the mathematical expression of the received $M$ th subsignal by ground device can be written as:

$$
Y^{(M)}(\omega)=N^{(M)}(\omega)+S(\omega) H^{(k)}(\omega)
$$

Where, $Y^{(M)}(\omega)$ is frequency expression of the signal of this path; $N^{(M)}(\omega)$ is the Gaussian white noise interference of this channel, with average value of 1 , and standard deviation of $0 ; S(\omega)$ is the emission signal, its time domain signal expression $s(t)$ can be determined by the following model:

$$
s(t)=\sum_{i=1}^{\infty} \sum_{j=0}^{N_{c}} a_{i} b_{j} A\left(t-i T_{s}-j T_{c}\right)
$$

Where, $T_{s}$ represents the symbol cycle of pulse signal ; $T_{c}$ represents the emission cycle of pulse signal ; $N_{c}$ represents the maximum frequency of occurrence of pulse symbol in $T_{c} ; b_{j}$ is the pseudorandom sequence in modulation process, and satisfies $b_{j} \in\{+1,-1\} ; a_{i}$ is pulse symbol sequence of modulation, and satisfies $a_{i} \in\{+1,-1\}$ 。

Here, each parameter in formula (3) also satisfies the expression as follows:

$$
A(t)=e^{\left(1-4 \pi t^{2} / \Lambda^{2}\right)\left(-2 \pi t^{2} / \Lambda^{2}\right)}
$$

Where, $\Lambda$ is step response of signal pulse.

According to above model, the model (2) can be evolved as:

$$
\begin{aligned}
& Y^{(M)}(\omega)=N^{(M)}(\omega)+S(\omega) H^{(k)}(\omega) \\
& =N^{(M)}(\omega)+S(\omega) \sum_{l=1}^{M} \beta_{l}^{(M)} e^{-j \omega \omega_{l}}
\end{aligned}
$$

When the ground device received the signal of model (5), and through the process of $\lambda$ uniformly-spaced sampling, its sampling frequency $\Delta \omega$ satisfies:

$$
\Delta \omega=2 \pi / \lambda
$$

The sampling signal of model (5) obtained after $\lambda$ uniformly-spaced sampling as:

$$
\begin{aligned}
& Y^{(M)}\left(\omega_{\lambda}\right)=N^{(M)}\left(\omega_{\lambda}\right)+S\left(\omega_{\lambda}\right) H^{(k)}\left(\omega_{\lambda}\right) \\
& =N^{(M)}\left(\omega_{\lambda}\right)+S(\omega) \sum_{l=1}^{M} \beta_{l}^{(M)} e^{-j \omega_{\lambda} \omega_{l}}
\end{aligned}
$$

Where, $\omega_{\lambda}=\lambda \Delta \omega$.

In order to facilitate calculation, the model (7) vector matrix can be simplified to:

$$
\begin{gathered}
y_{m}=w_{m}+\beta_{m} E_{\omega} S \\
\beta_{m}=\left[\beta_{1}^{(\lambda)}, \beta_{2}^{(\lambda)}, \ldots ., \beta_{\lambda}{ }^{(\lambda)}\right]^{T} \\
w_{m}=\left[w^{(\lambda)}\left(\omega_{1}\right), w^{(\lambda)}\left(\omega_{2}\right), \ldots ., w^{(\lambda)}\left(\omega_{\lambda}\right)\right]^{T}
\end{gathered}
$$

$y_{m}=\left[Y^{(M)}\left(\omega_{1}\right), Y^{(M)}\left(\omega_{2}\right), \ldots \ldots \ldots, Y^{(M)}\left(\omega_{\lambda}\right)\right]^{T}$ represents sampling signal of receiving signal obtained by $\lambda$ uniformly-spaced sampling ; $\beta_{m}$ represents the fading vector of subsignal of $M$ th path; $w_{m}$ represents the sampling signal of gaussian white noise obtained by $\lambda$ uniformly-spaced sampling ; $S=\Lambda\left[S\left(\omega_{1}\right), S\left(\omega_{2}\right), \ldots \ldots, S\left(\omega_{\lambda}\right)\right]^{T}$ is the diagonal matrix of rank value $\lambda$, the elements on diagonal is the sampling value of 
$S(\omega)$ after $\lambda$ uniformly-spaced sampling ; $E_{\omega}$ is the unit time delay matrix, $E_{\omega}=\left[E_{1}, E_{2}, \ldots ., E_{i}\right]$, where $E_{i}$ satisfies :

$$
E_{i}=\left[1, e^{-j \Delta \omega \omega_{i}}, e^{-2 j \Delta \omega \omega_{i}}, \ldots \ldots, e^{-\lambda j \Delta \omega \omega_{i}}\right]^{T}
$$

\section{Parameter Estimation of Signal Receiving Spectrum}

\subsection{The Structure of Receiving and Receiving Spectrum Function of Signal}

Due to the data source of positioning signal is space-based platform, such as the typical Beidou satellite orbit height is $36,000 \mathrm{~km}$, and the antenna distance of receiving device can be neglected when compared with the height of satellite orbit, so the injection direction of each path of signal is parallel.

Set $M$ sub-signals are the $M$ antenna reception, respectively, the received signals are $Y_{1}, Y_{2}, \ldots \ldots ., Y_{M}$; The corresponding time delay is $E_{i}$, where $\mathrm{i}=1,2,3 \ldots \ldots . . \mathrm{M}$. According to the model (8) can get the following equations:

$$
\begin{gathered}
Y_{1}=w_{1}+\beta_{1} E_{\omega_{1}} S \\
Y_{2}=w_{2}+\beta_{2} E_{\omega_{2}} S \\
\ldots \ldots . . \\
Y_{m}=w_{m}+\beta_{m} E_{\omega_{m}} S
\end{gathered}
$$

Where, $S=\Lambda\left[S\left(\omega_{1}\right), S\left(\omega_{2}\right), \ldots \ldots, S\left(\omega_{\lambda}\right)\right]^{T}$ is the diagonal matrix with matrix rank value of $\lambda$, the elements on diagonal is the sampling value after $\lambda$ uniformly-spaced sampling of $S(\omega), \quad E_{\omega_{i}}(\mathrm{i}=1,2, \ldots ., \mathrm{M})$ is the unit time delay matrix, and stasfies :

$$
\begin{aligned}
E_{\omega_{1}} & =\left[\begin{array}{cccc}
1 & 1 & \ldots & 1 \\
e^{\left.-j \Delta \omega \omega^{(1)}\right)_{1}} & e^{-j \Delta \omega \omega^{(2)}{ }_{1}} & & e^{-j \Delta \omega \omega^{(\lambda)}{ }_{1}} \\
\vdots & \vdots & \ddots & \vdots \\
e^{-\lambda j \Delta \omega \omega^{(1)}{ }_{1}} & e^{\left.-\lambda j \Delta \omega \omega^{(2)}\right)_{1}} & \ldots & e^{\left.-\lambda j \Delta \omega \omega^{(\lambda)}\right)_{1}}
\end{array}\right] \\
E_{\omega_{2}} & =\left[\begin{array}{cccc}
1 & 1 & \ldots & 1 \\
e^{-j \Delta \omega \omega^{(1)}{ }_{2}} & e^{\left.-j \Delta \omega \omega^{(2)}\right)_{2}} & & e^{-j \Delta \omega \omega^{(\lambda)_{2}}} \\
\vdots & \vdots & \ddots & \vdots \\
e^{-\lambda j \Delta \omega \omega^{(1)}{ }_{2}} & e^{\left.-\lambda j \Delta \omega \omega^{(2)}\right)_{2}} & \ldots & e^{-\lambda j \Delta \omega \omega^{(\lambda)_{2}}}
\end{array}\right] \\
E_{\omega_{m}} & =\left[\begin{array}{cccc}
1 & 1 & \ldots & 1 \\
e^{\left.-j \Delta \omega \omega^{(1)}\right)_{m}} & e^{\left.-j \Delta \omega \omega^{(2)}\right)_{m}} & & e^{-j \Delta \omega \omega^{(\lambda)_{m}}} \\
\vdots & \vdots & \ddots & \vdots \\
e^{-\lambda j \Delta \omega \omega^{(1)}{ }_{m}} & e^{-\lambda j \Delta \omega \omega^{(2)}{ }_{m}} & \ldots & e^{-\lambda j \Delta \omega \omega^{(\lambda)}{ }_{m}}
\end{array}\right]
\end{aligned}
$$

Set $\Delta t_{i, j}=t_{i}-t_{j}$ as the arriving time difference of any ith antenna and jth antenna, the included angle $\theta$ of arriving direction of signal in figure 1 and normal is the signal wave arriving direction DOA. So $\Delta t_{i, j}$ is :

$$
\Delta t_{i, j}=d_{i, j} \sin \theta / c
$$

Where, $d_{i, j}$ is the distance between ith antenna and jth antenna, $C$ is the transmission speed of electromagnetic wave in vacuum.

According to above model, then estimation $\bar{\theta}_{i, j}$ of DOA satisfied:

$$
\bar{\theta}_{i, j}=\arcsin \left(c \Delta t_{i, j} / d\right)
$$

Obviously, it can be known from model (16) (17), the more precision of the TOA estimation, and DOA estimation is more precision.

Therefore, for sub-signal of any ith path and jth path, this paper structures incidence matrix $D_{i, j} \in \Omega^{2 M \times M}$, its model as 


$$
\begin{gathered}
D_{i, j}=\left[\begin{array}{c}
Y_{i} \\
Y_{j}
\end{array}\right]=\left[\begin{array}{c}
w_{i} \\
w_{j}
\end{array}\right]+\left[\begin{array}{c}
S E_{\omega_{i}} \\
S E_{\omega_{j}}
\end{array}\right] B \\
\text { if } P\left(t_{i}, t_{j}\right)=\left[\begin{array}{l}
S E_{\omega_{i}} \\
S E_{\omega_{j}}
\end{array}\right] \in \Omega^{2 M \times \lambda}, \quad w(i, j)=\left[\begin{array}{c}
w_{i} \\
w_{j}
\end{array}\right] \in \Omega^{2 M \times M}, \quad \text { then formula (18) can be evolved as: } \\
D_{i, j}=w(i, j)+P\left(t_{i}, t_{j}\right) B
\end{gathered}
$$

Then set $\bar{C}=D_{i, j} D_{i, j}{ }^{H}, \quad D_{i, j}{ }^{H}$ as the conjugate transpose of $D_{i, j}$, obviously, $\bar{C}$ has $2 M$ characteristic values. And decompose $\bar{C}$ according to characteristic value :

$$
\bar{C}=\overline{M_{s}}+\overline{N_{s}}
$$

Where, $\overline{M_{s}} \in \Omega^{2 M \times \lambda}$ is signal space; $\overline{N_{s}} \in \Omega^{2 M \times(2 M-\lambda)}$ is the Gaussian white noise space.

So Gaussian white noise is mutually independent with receiving signal, so $\bar{M}_{s} \in \Omega^{2 M \times \lambda}$ is mutually orthogonal with $\bar{N}_{s} \in \Omega^{2 M \times(2 M-\lambda)}$. Therefore, the column vector of $\bar{C}$ is also mutually orthogonal with $\bar{N}_{s} \in \Omega^{2 M \times(2 M-\lambda)}$, so the receiving spectral function $F(i, j)$ as :

$$
F(i, j)=\frac{1}{p\left(t_{i}, t_{j}\right)^{H}{\overline{N_{s}}}_{N_{s}}^{H} p\left(t_{i}, t_{j}\right)}
$$

Where, $p\left(t_{i}, t_{j}\right)$ is the column vector of $P\left(t_{i}, t_{j}\right), \quad p\left(t_{i}, t_{j}\right)^{H}$ and ${\overline{N_{s}}}^{H}$ are conjugate transposes of $p\left(t_{i}, t_{j}\right)$ and $\bar{N}_{s}$ respectively.

By peak searching of $F(i, j)$ in signal space, it obtains the maximum TOA estimation of any ith path and jth path of sub-signals.

\subsection{TOA Estimation Based On Receiving Spectrum Function}

$F(i, j)$ in model $(21)$ is the received spectrum function of any two paths of the signals receiving at the same time. In fact multiple paths of signals based on the low intensity of nanosecond wireless network are mutually orthogonal and independent of each other, thus it can perform receiving spectrum estimation on any path of signals, respectively.

For sub-signal i of any path, its received signal can be written as the corresponding $Y_{i}$ form of formula(12). Let $\bar{G}=Y_{i} Y_{i}{ }^{H}$, where $Y_{i}{ }^{H}$ is the conjugate transpose of $Y_{i}$, obviously, $\bar{G}$ has $2 M$ characteristic values, decompose $\bar{G}$ according to characteristic value into the weight of signal subspace and Gaussian white noise subspace :

$$
\bar{G}=\overline{M_{s i}} \Lambda{\overline{M_{s i}}}^{H}+\overline{N_{s i}} \Lambda{\overline{N_{s i}}}^{H}
$$

Where, $\overline{\boldsymbol{M}_{s i}}$ is signal subspace ; $\overline{N_{s i}}$ is Gaussian white noise subspace ; ${\overline{\boldsymbol{M}_{s i}}}^{H}$ and ${\overline{N_{s i}}}^{H}$ represent the corresponding conjugate transpose ; $\Lambda$ is diagonal matrix.

According to model (18), $\overline{M_{s i}}=S E_{\omega i}$. It obvious that the column vector of $S E_{\omega i}$ is mutually orthogonal with the corresponding vector of $\overline{N_{s i}}$, then receiving spectral function $f_{i}$ of sub-signal $i$ of any path can be written as :

$$
f_{i}=\frac{1}{\left[s e_{\omega_{i}}\right]^{H} \overline{N_{s i}}{\overline{N_{s i}}}^{H} s e_{\omega_{i}}}
$$

Where, $e_{\omega i}$ is the column vector of $E_{\omega i}$.

then according to model (15) we have:

$$
e_{\omega_{i}}=\left[1, e^{-j \Delta \omega \omega^{(i)}}, e^{-2 j \Delta \omega \omega^{(i)}{ }_{m}}, \ldots \ldots ., e^{-\lambda j \Delta \omega \omega^{(i)}{ }_{m}}\right]
$$

By searching the corresponding maximum of $f_{i}$, that is the maximum TOA estimation of any ith path sub-signal. Let $z=e^{-j \Delta \omega \omega^{(i)}}{ }_{m}$, then the complex expression form of formula (23) as shown below :

$$
f_{i}(z)=\frac{1}{[s f(z)]^{H}{\overline{N_{s i}}}_{N_{s i}}^{H} s f(z)}
$$




$$
f(z)=\left[1, z^{-1}, z^{-2}, \ldots ., z^{-m}\right]
$$

It can be known from complex theory[7-8], pole polynomial $\bar{f}(z)$ of model (25) as

$$
\bar{f}(z)=[s f(z)]^{H} \bar{N}_{s i} \bar{N}_{s i}{ }^{H} s f(z)
$$

Due to model (27) has a total of $2 M$ complex roots on complex domain, and is symmetrical distribution on the unit circle ; And the dimension of the signal subspace is $2 M-\lambda$. Therefore, complex root of $\bar{f}(z)$, according to the distance close to unit circle, selects the front $2 M-\lambda$ complex root $\bar{z}_{i}(\mathrm{i}-1,2, .$.$) as TOA estimation \bar{t}_{i}$ :

$$
\bar{t}_{i}=\frac{1}{2 M-\lambda} \sum_{i=1}^{2 M-\lambda} \bar{z}_{i}
$$

Due to DOA estimation requires compassion of two paths of signals, so according to model (18),

The corresponding incidence matrix $D_{i, j}$ of any two path of signal I and $\mathrm{j}$ can be obtained. Then let $\bar{C}=D_{i, j} D_{i, j}{ }^{H}$, the model (20) can be formed. Where, $\bar{M}_{s} \in \Omega^{2 M \times 2}$ is signal space, $\quad \bar{N}_{s} \in \Omega^{2 M \times(2 M-\lambda)}$ is Gaussian White noise space.

Because of Gaussian white noise and receiving signals are mutually independent, therefore, $\overline{M_{s}} \in \Omega^{2 M \times \lambda}$ is mutually orthogonal with $\overline{N_{s}} \in \Omega^{2 M \times(2 M-\lambda)}$, so column vector of $\bar{C}$ is mutually orthogonal with $\overline{N_{s}} \in \Omega^{2 M \times(2 M-\lambda)}$. Its intersection cost function $H(i, j)$ as

$$
H(i, j)=p\left(t_{i}, t_{j}\right)^{H}{\overline{N_{s}}}_{N_{s}}^{H} p\left(t_{i}, t_{j}\right)
$$

Where, the $p\left(t_{i}, t_{j}\right) 、 \bar{N}_{s}$ and model (21)are the same.

Compared the expression of function $h(i, j)$ as follows

$$
h(i, j)=\max _{i, j} H(i, j)
$$

Where, $i, j=1,2,3 \ldots ., M$

Through calculate maximum cost function $h(i, j)$, that is the optimal arriving time, then according model (17) to obtain the optimal DOA estimation.

\subsection{TOA and DOA Parameter Estimation Method}

When ground receiving device receives a signal, you first need to calculate the analytical expression of the various independent sub-signal, and then on the basis of analytical expression to obtain the covariance matrix of sub-signal space, and conduct signal subspace and noise subspace decomposition to the matrix, in order to get characteristic value and characteristic vector, and then perform characteristic value and characteristic vector estimation and according to this. When each path signal is calculated according to the process, start the comparison process, and substitute it into model (29) for comparison to get TOA estimation. Then, it obtains the DOA estimation through pairwise comparison. Take the maximum value of TOA estimation and DOA estimation, that is TOA estimation and DOA estimation of the whole signal. The constructing steps of the whole estimation method as follows:

Step 1 : Receiving sub-signal, to detect sub-signal whether can be sample processing or not, and obtain the analytical expression $Y_{i}$ of sub-signal of ith path 【model (12)】;

Step $2:$ then structure covariance matrix $\bar{G}$ of $Y_{i}$, and solve its characteristic value;

Step 3 : Then perform zero solving of complex equation of model (25);

Step $4:$ according to model (28), after the TOA estimation of sub-signal of ith path is obtained, the rest sub-signal of $M-1$ path can be obtained according to Step 1 to Step 3 successively, and obtain the corresponding TOA estimation, select the maximum of model (30) as the optimal TOA estimation of the system; 
Step 5 : Substitute the optimal TOA estimation into model (16), and solve the optimal DOA estimation;

Step 6 : After this cycle complete, it waits the next cycle of transmission.

\section{Simulation Experiment}

Based on the NS2 simulation platform, this paper carried out simulation on the proposed estimation methods, using the formula (3) to generate the receive time-domain signal. The simulation parameters are shown in table 1 below:

Table 1. Simulation Parameters

\begin{tabular}{cc}
\hline Parameter & Number \\
\hline$\left(T_{s}\right)$ duration of the signal cycle & $600 \mathrm{~ms}$ \\
$(f)$ Satellite positioning signal frequency & $1.575 \mathrm{MHZ}$ \\
$(m)$ background noise average value & 0 \\
$\left(\sigma^{2}\right)$ Background noise variance & 1 \\
Initial phase of signal & 0 \\
$(\mathrm{~dB})$ receiver receiving SNR & $-128 \mathrm{db}$ \\
input signal strength of receiver & \\
Signal accumulation time & $600 \mathrm{~ms}$ \\
signal positioning times & 10000 \\
signal offset frequency & $2000 \mathrm{HZ}$ \\
sub-signal paths & no more than 64 paths \\
\hline
\end{tabular}

To verify the excellent of the proposed estimation method, this paper set PM algorithm [10], ESPRIT algorithm[11] as control group. The proposed method compared the TOA and DOA estimation precision with control group. In order to make comparison in scale, it generated simulation environment according to the simulation parameters shown in table 1 .

Figure 1 showed the test of proposed method on TOA estimation precision under different sub-signal paths. It can be seen from the figure that, with the increasing of subsignal paths, the TOA precision of the proposed method was also increasing constantly. This was because the proposed method introduced space deconstruction way, which decomposed the received signal into signal subspace and noise subspace, then carried on characteristic value assessment and zero calculation, with the increase of sub-signal paths, the number of evaluation was also increased constantly, so the TOA precision was also improved correspondingly. 


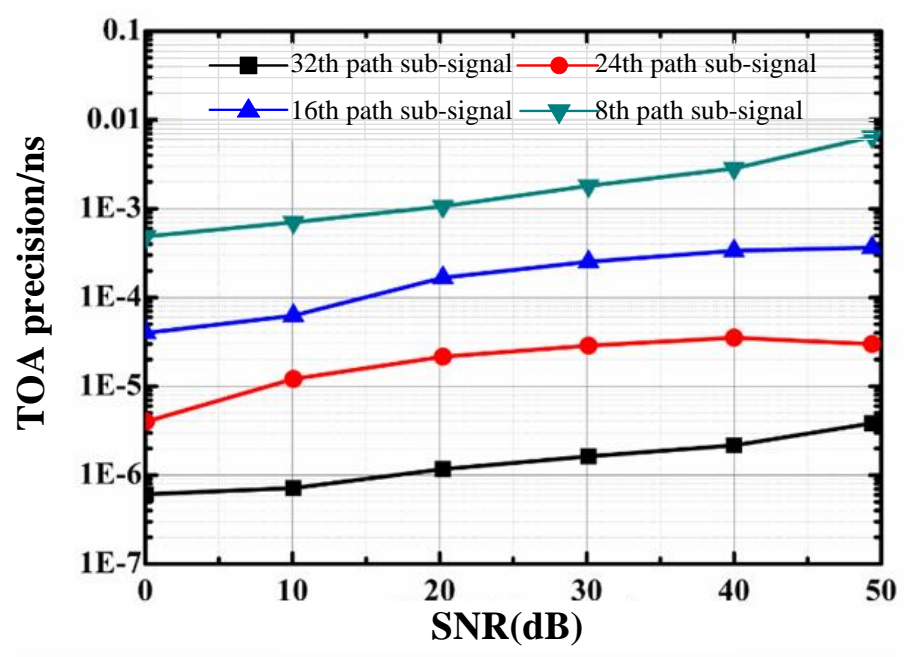

\section{Figure 1. TOA Parameter Precision Estimation Test Under Different Sub- signal Paths}

Figure 2 showed the test of proposed method on DOA estimation precision under different sub-signal paths. It can be seen from the figure that, with the increasing of subsignal paths, the DOA precision of the proposed method was also increasing constantly, this was because the DOA precision was associated with TOA precision between different sub-signals, with the increase of the path, the TOA precision difference between different sub-signals was also increasing, at last, by constantly comparison of DOA precision of the signal, therefore, the DOA precision was improved accordingly.

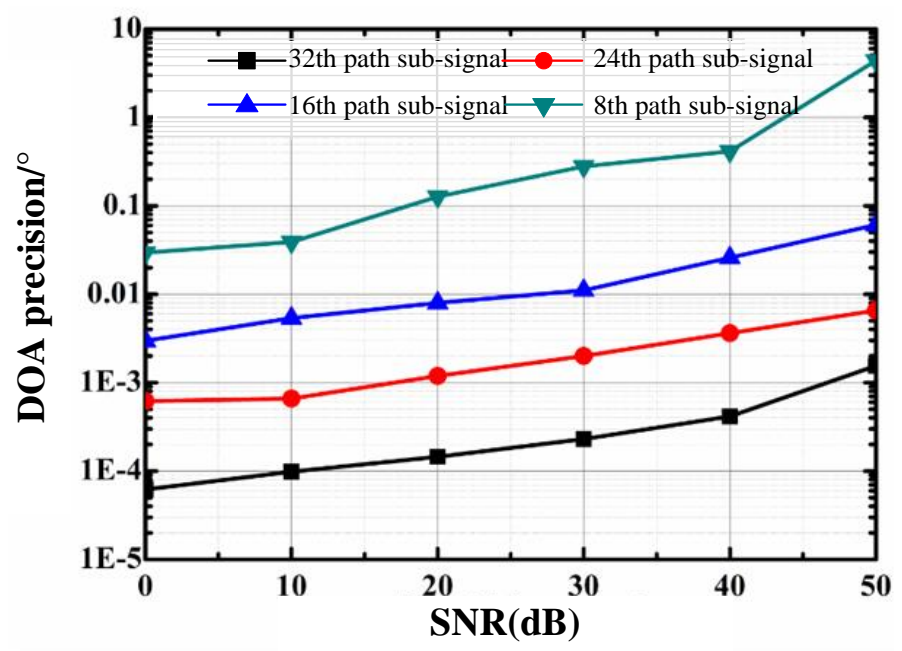

Figure 2. DOA Parameter Precision Estimation Test under Different Subsignal Paths

Figure 3 showed the test results of proposed method, PM algorithm, ESPRIT algorithm on TOA estimation precision under the different receiver SNR intensity. It can be seen from the figure that, with the increasing of the receiver SNR, the precision of three methods were all falling; but the gap of the precision between PM algorithm and ESPRIT algorithm was constantly expanding. Reason was that the PM, ESPRIT algorithm did not introduce contrast mechanism when considering multipath comparison; while the proposed method carried on analysis of received signal, the signal was resolved into signal subspace and noise subspace, and both maintained in orthogonal state was 
guaranteed, thus the interference of background noise was reduced, and the precision of the estimation was improved.

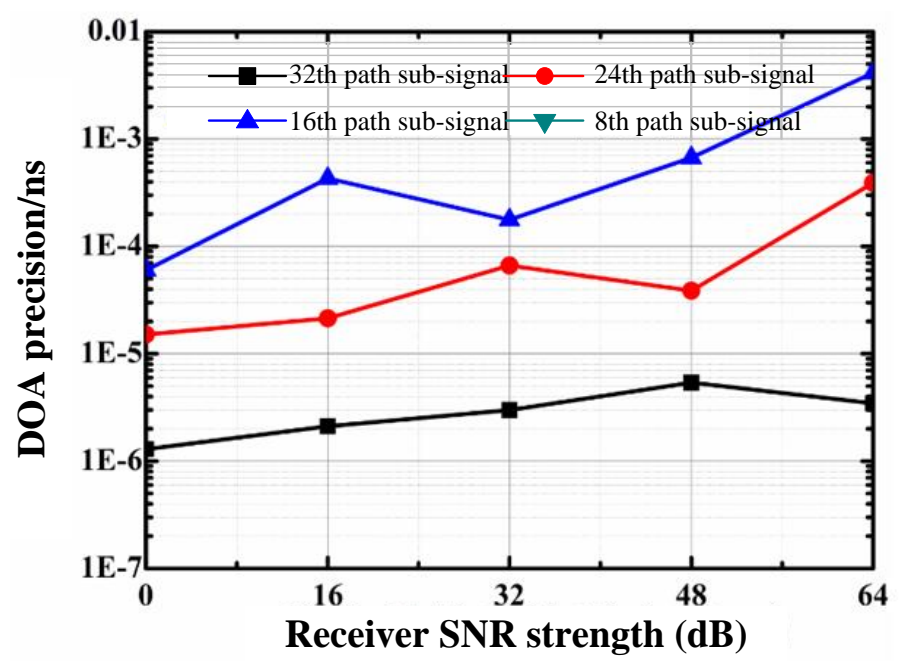

Figure 3. Test Results of Three TOA Precision Estimation Methods under Different Receiver SNR Strength

Figure 4 showed the estimation precision comparison of proposed method, PM algorithm, ESPRIT algorithm on DOA under different intensity of receiver SNR. It can be seen from the figure that, with the increasing of the receiver SNR, the difference of precision of the three on DOA was widening, this is because the proposed method adopted the contrast and zero calculation mechanism, which made DOA and TOA precision of the received signal was not a direct linear relationship, with the increasing of TOA precision, DOA precision showed faster increase.

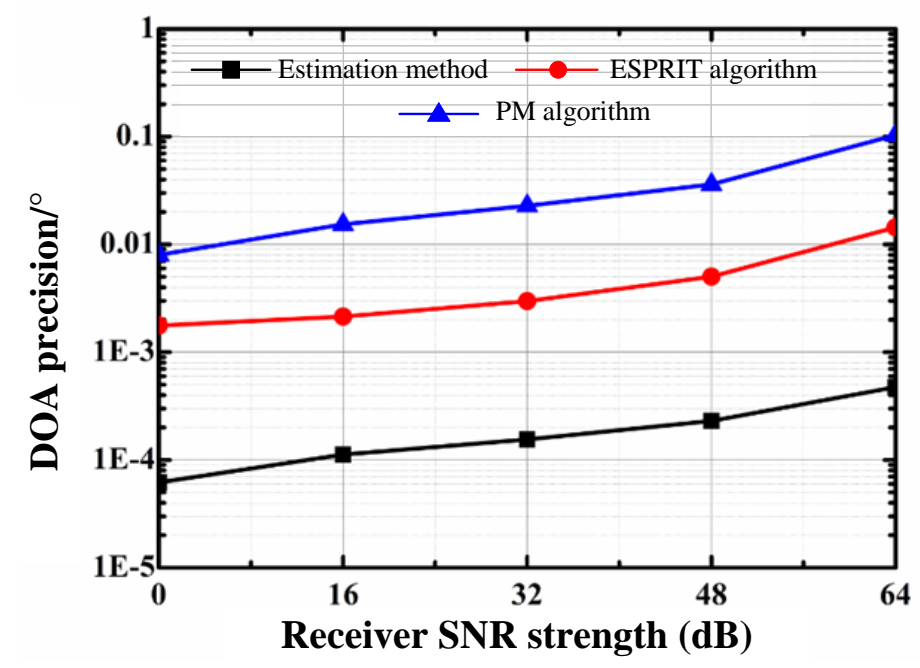

Figure 4. Test Results of Three DOA Precision Estimation Methods under Different Receiver SNR Strength

Figure 5, Figure 6 showed the estimation precision comparison of proposed method, PM algorithm, ESPRIT algorithm on TOA and DOA under different background noise intensity, it can be seen from the figures that, with the increasing of background noise intensity, while the accuracy of the proposed method in this paper was declining, but the 
amplitude was small, shown a steady state; the accuracy of PM algorithm, ESPRIT algorithm presented the trend of declining. This was because with the increasing of background noise intensity, the proportion of power of background noise in received signal was also increasing, leading to the accuracy of PM algorithm, ESPRIT algorithm reduced due to the effects of noise. And the proposed method introduced orthogonal mechanism, whatever the strength of the background noise, noise and signal remained the orthogonal state, and the precision of the proposed method in this paper was improved.

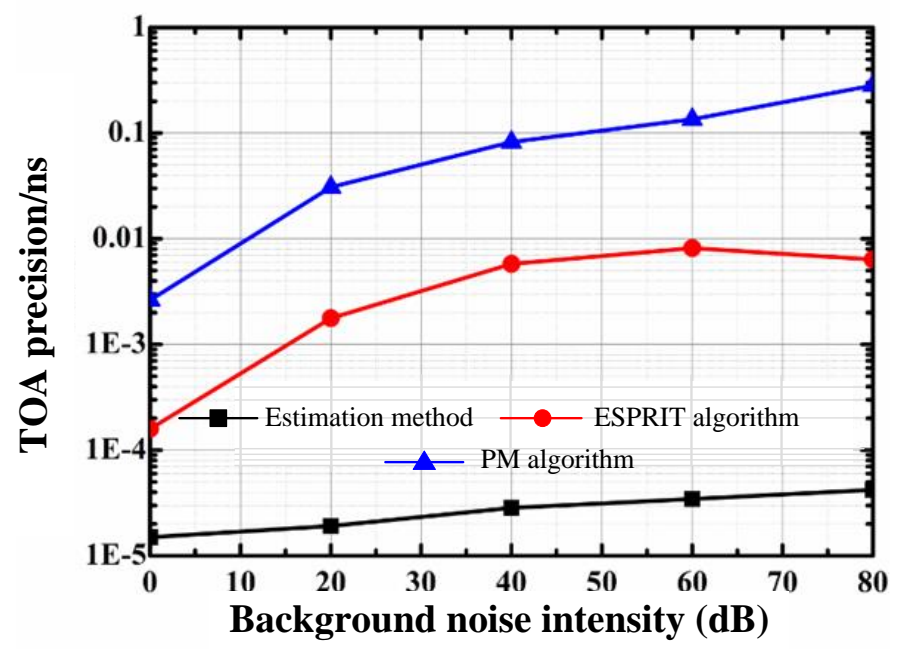

Figure 5. Test Results of Three TOA Precision Estimation Methods under Different Intensity Of Background SNR

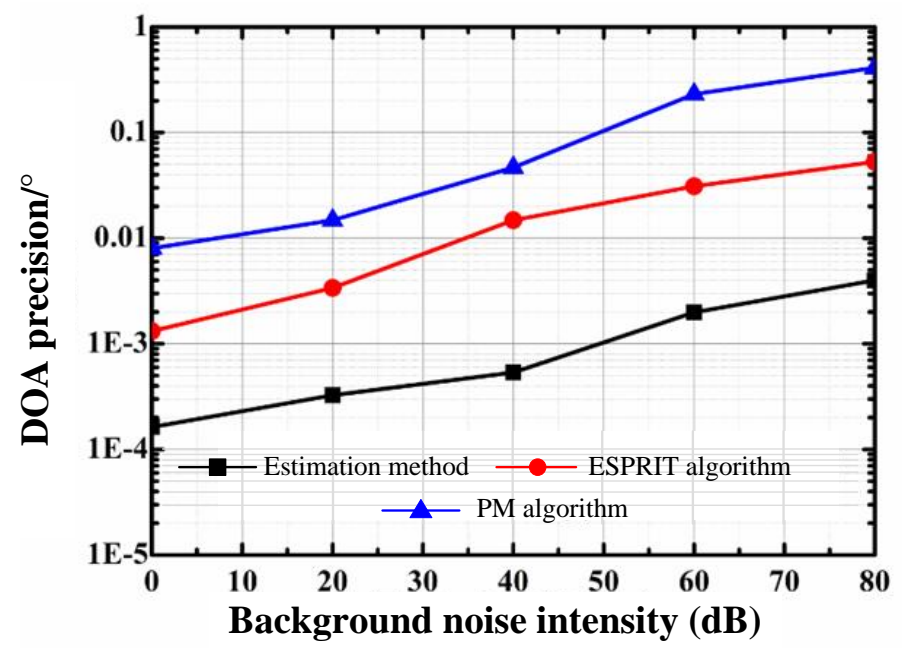

Figure 6. Test Results of Three DOA Precision Estimation Methods under Different Intensity Of Background SNR

\section{Conclusion}

For the TOA and DOA estimation precision on subnanosecond of current network signal was not high, and difficult to resist noise effectively, this paper put forward a new wireless network signal receiving spectrum to obtain parameter estimation method. By way of sampling, sampling the launch signals as multidimensional independent subsignal; then according to digital characteristics of the signal (characteristic value and characteristic vector) to perform segmentation again, and formed the mutually orthogonal 
signal space and noise space, so the effective zero in complex domain is implemented, thus the estimation precision was greatly improved. Simulation results showed that, compared with PM algorithm, ESPRIT algorithm, the TOA and DOA estimation precision of proposed method was higher, with better ability of noise resistance.

\section{Acknowledgement}

The research is supported by Chongqing Municipal Education Commission science and technology research project (KJ132202).

\section{References}

[1] D. Jiang, Z. Xu and Z. Lv, "A multicast delivery approach with minimum energy consumption for wireless multi-hop networks", Telecommunication Systems, (2015), pp. 1-12.

[2] Y. Lin, J. Yang and Z. Lv, "A self-assessment stereo capture model applicable to the internet of things", Sensors, vol. 15, no. 8, (2015), pp. 20925-20944.

[3] Y. Liang, "Satisfaction With Economic and Social Rights and Quality of Life in a Post-Disaster Zone in China", Evidence From Earthquake-Prone Sichuan, Disaster Medicine and Public Health Preparedness, vol. 9, no. 2, pp. 111-118.

[4] Y. Liang, "Correlations Between Health-Related Quality of Life and Interpersonal Trust", Comparisons Between Two

[5] Generations of Chinese Rural-to-Urban Migrants, Social Indicators Research, vol. 123, no. 3, pp. 677700 .

[6] Y. Liang and P. Lu, "Medical insurance policy organized by Chinese government and the health inequity of the elderly", longitudinal comparison based on effect of New Cooperative Medical Scheme on health of rural elderly in 22 provinces and cities, International Journal for Equity in Health.

[7] Y. Lianga and D. Zhu, "Subjective Well-Being of Chinese Landless Peasants in Relatively Developed Regions: Measurement Using PANAS and SWLS", Social Indicators Research, vol. 123, no. 3, pp. 817835.

[8] Y. Liang and X. Wang, "Developing a new perspective to study the health of survivors of Sichuan earthquakes in China", a study on the effect of post-earthquake rescue policies on survivors' healthrelated quality of life, Health Research Policy and Systems.

[9] J. Hu and Z. Gao, "Modules identification in gene positive networks of hepatocellular carcinoma using Pearson agglomerative method and Pearson cohesion coupling modularity", Journal of Applied Mathematics, (2012).

[10] Y. Geng, J. Chen, R. Fu, G. Bao and K. Pahlavan, "Enlighten Wearable Physiological Monitoring systems: On-Body RF Characteristics Based Human Motion Classification Using a Support Vector Machine", PP(99), (2015), pp. 1-16.

[11] X. Song and Y. Geng, "Distributed Community Detection Optimization Algorithm for Complex Networks", Journal of Networks, vol. 9, no. 10, (2014), pp. 2758-2765.

[12] K. Pahlavan, P. Krishnamurthy and Y. Geng, "Localization Challenges for the Emergence of the Smart World", Access, IEEE, vol. 3, no. 1, (2015), pp. 1-11.

[13] J. He, Y. Geng, Y. Wan, S. Li and K. Pahlavan, "A cyber physical test-bed for virtualization of RF access environment for body sensor network. Sensors Journal, IEEE, vol. 13, no. 10, (2013), pp. 38263836.

[14] Z. Lv, A. Teka and S. Da, "Game on, science-how video game technology may help biologists tackle visualization challenges", PloS one, vol. 8, no, 3, (2013), p. 57990.

[15] T. Su, W. Wang and Z. Lv, "Rapid Delaunay triangulation for randomly distributed point cloud data using adaptive Hilbert curve", Computers \& Graphics, vol. 54, (2016), pp. 65-74.

[16] J. Hu, Z. Gao and W. Pan, "Multiangle Social Network Recommendation Algorithms and Similarity Network Evaluation", Journal of Applied Mathematics, (2013).

[17] S. Zhou, L. Mi, H. Chen and Y. Geng, "Building detection in Digital surface model", 2013 IEEE International Conference on Imaging Systems and Techniques (IST), (2012).

[18] J. He, Y. Geng and K. Pahlavan, "Toward Accurate Human Tracking: Modeling Time-of-Arrival for Wireless Wearable Sensors in Multipath Environment", IEEE Sensor Journal, vol. 14, no. 11, (2014), pp. 3996-4006. 


\section{Author}

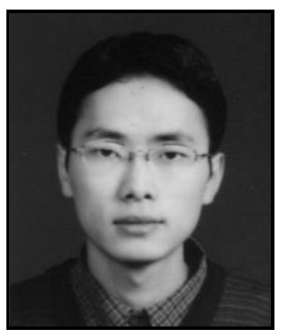

Shao Youwei, he is an Associate Professor, Chongqing College of Electronic Engineering. Born in 1979, Mr. Shao received master degree from Chongqing University, and his main research interests are Sensing and Control. He has published several research papers in scholarly journals in the above research areas. 
International Journal of Future Generation Communication and Networking Vol. 9, No.10, (2016) 